\title{
Robotic Surgical Training in an Academic Institution
}

W. Randolph Chitwood, Jr., MD, L. Wiley Nifong, MD, William H. H. Chapman, MD, Jason E. Felger, MD, B. Marcus Bailey, MD, Tara Ballint, MD, Kim G. Mendleson, MD, PhD, Victor B. Kim, MD, James A. Young, MD, and Robert A. Albrecht, MD

From the Department of Surgery, Center for Minimally Invasive and Robotic Surgery, Brody School of Medicine, East Carolina University, Greenville, North Carolina

\section{Objective}

To detail robotic procedure development and clinical applications for mitral valve, biliary, and gastric reflux operations, and to implement a multispecialty robotic surgery training curriculum for both surgeons and surgical teams.

\section{Summary Background Data}

Remote, accurate telemanipulation of intracavitary instruments by general and cardiac surgeons is now possible. Complex technologic advancements in surgical robotics require well-designed training programs. Moreover, efficient robotic surgical procedures must be developed methodically and safely implemented clinically.

\section{Methods}

Advanced training on robotic systems provides surgeon confidence when operating in tiny intracavitary spaces. Three-dimensional vision and articulated instrument control are essential. The authors' two da Vinci robotic systems have been dedicated to procedure development, clinical surgery, and training of surgical specialists. Their center has been the first United States site to train surgeons formally in clinical robotics.

\section{Results}

Established surgeons and residents have been trained using a defined robotic surgical educational curriculum. Also, 30 multispecialty teams have been trained in robotic mechanics and electronics. Initially, robotic procedures were developed experimentally and are described. In the past year the authors have performed 52 robotic-assisted clinical operations: 18 mitral valve repairs, 20 cholecystectomies, and 14 Nissen fundoplications. These respective operations required 108, 28, and 73 minutes of robotic telemanipulation to complete. Procedure times for the last half of the abdominal operations decreased significantly, as did the knot-tying time in mitral operations. There have been no deaths and few complications. One mitral patient had postoperative bleeding.

\section{Conclusion}

Robotic surgery can be performed safely with excellent results. The authors have developed an effective curriculum for training teams in robotic surgery. After training, surgeons have applied these methods effectively and safely.
Surgeons always have sought methods to develop new operations, but many times have been limited by technology. In many instances, initial endoscopic surgical training of senior surgeons and residents alike proceeded along

Presented at the 121st Annual Meeting of the American Surgical Association, April 26-28, 2001, the Broadmoor Hotel, Colorado Springs, Colorado

Supported in part by educational grants from the Doris Duke Charitable Trust and the United States Surgical Corporation, Inc.

Correspondence: W. Randolph Chitwood, Jr., MD, Professor and Chairman, Department of Surgery, East Carolina University School of Medicine, Moye Boulevard, Greenville, NC 27858.

E-mail: chitwoodw@mail.ecu.edu

Accepted for publication April 26, 2001. variable pathways without significant prior procedure development or detailed curricula. Early clinical training frequently was at the expense of the best surgical results. Bonchek, ${ }^{1}$ Lytle, ${ }^{2}$ and Cooley ${ }^{3}$ have cautioned surgeons who veer from established techniques with proven results, even if much larger incisions are required.

Multispecialty procedure development is very important when any new technology is introduced in surgery. Our trek for developing surgical robotics and training surgeons has been predicated on quality expected from conventional procedures, or "base camps." Progression to each successive level has been followed by technologic "acclimatization" and experience before attempting the last challenge to surgical telemanipulation. ${ }^{4-8}$ Complex endoscopic reconstruc- 


\section{Table 1. ROBOTIC SURGICAL TRAINING CURRICULUM LEVELS}

I. Didactic overview

- Understand robotic vision and electronics

- Understand robotic instrumentation

- Understand robotic ergonomics

- Understand robotic limitations

II. Inanimate laboratory

- Master operative console

- Master robotic operative cart

- Master instrument and camera control

III. Animal laboratory

- Console surgeon-master suturing, tissue cutting, suture tying

- Patient-side assistant-master:

o Instrument exchanges

- Camera cleaning

- Cauterization

- Clip application

- Retraction

- Trocar positioning

IV. Cadaver laboratory

- Master trocar positioning

- Apply I-III to human anatomy

- Apply I-III to variable body habitus

V. Operative observation

- Determine differences from I-IV

- Observe interaction with adjunctive surgical technology

tive operations require three-dimensional vision, articulated intracavitary instrumentation, and telemanipulation. To expand these new capabilities, robotic surgical procedures were developed at East Carolina University (ECU), and objective-based curriculum levels (Table 1) were designed to optimize surgeon and team training, which is necessary to attain the best early clinical results.

\section{METHODS}

\section{Robotic Instrumentation}

The ECU robotic training program has been based on two dedicated da Vinci surgical systems (Intuitive Surgical, Inc., Mountain View, CA). To optimize training and clinical emulation, similar environments were created in the laboratory and patient operating room. The surgical robot has an articulated "endowrist" at the end of two (7-mm outer diameter) surgical arms (Fig. 1). ${ }^{9}$ This device permits intracavitary manipulation of various 2- to 4-mm instrument tips through seven degrees of excursion, emulating the human wrist. The surgeon operates from a master console using a $10 \times$ three-dimensional, wide-angle camera $(1-\mathrm{cm}$ outer diameter) to effect simultaneous exact intracavitary movements of tiny articulated instruments. For clinical cases, our operating console is placed 10 feet from the patient's side, providing continuous surgeon control of both the surgical environment and the internal operation. A patient-side robotic cart positions and drives the wristlike devices, while an assistant adjusts and performs instrument exchanges. Operators essentially become immersed in the surgical landscape, which helps to effect a "telepresence" with optimal access and dexterity. With experience surgeons develop "near" proprioception, but thus far tactile (haptic) feedback remains attenuated significantly. Robotic arms and "wrist" instruments are placed through ports and converge in the surgical field. Seven degrees of motion freedom are provided by the combination of trocar-positioned arms (insertion, pitch, yaw) and articulated instrument wrists (yaw, pitch, roll, and grip). From the operating console, full $x, y$, and $z$ axis agility is affected by coordinating foot pedal clutching and hand-motion sensors. Con-

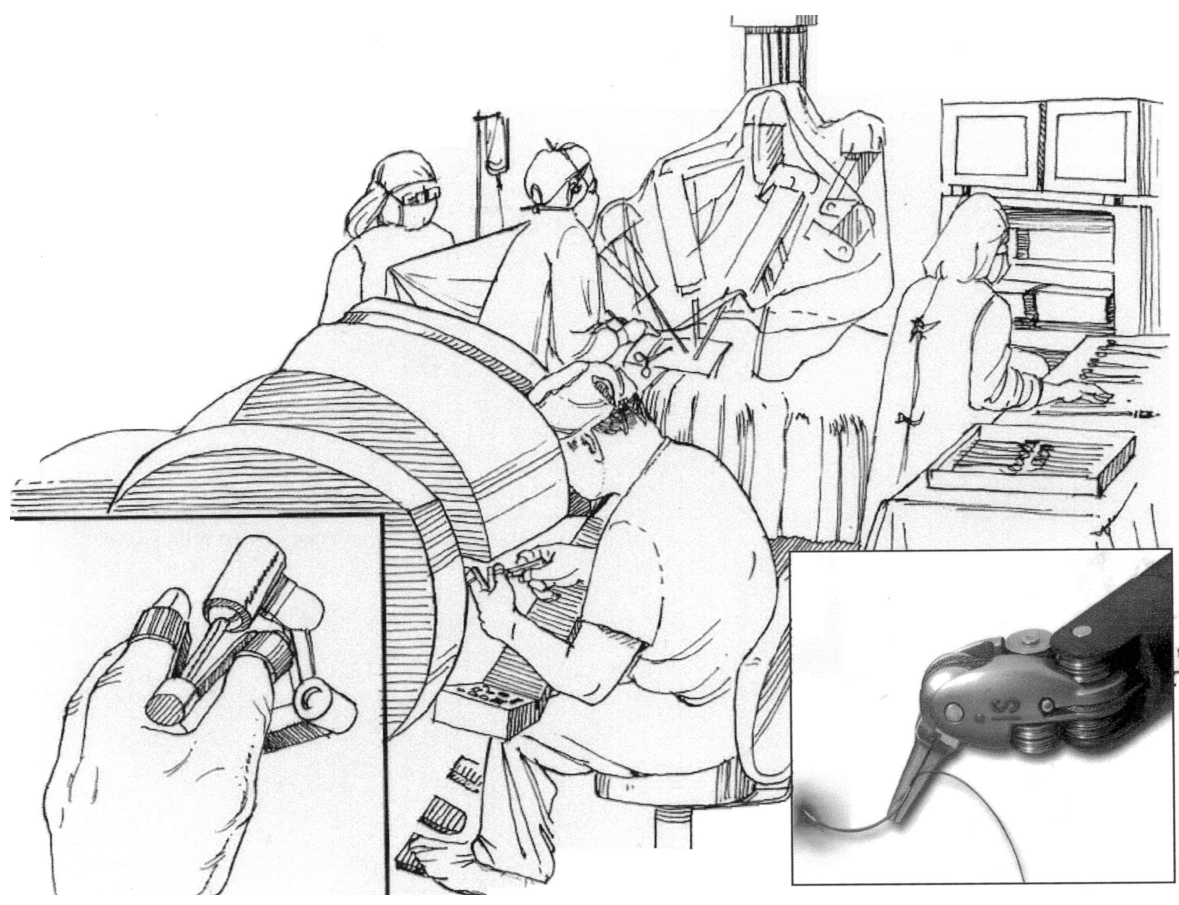

Figure 1. The surgeon's console is placed 10 feet from the patient's side. A patientside surgical cart positions and drives the articulating instruments while an assistant facilitates the procedure and performs instrument exchanges. Surgeons essentially become immersed in the surgical landscape. (Inset) The surgical endowrist provides seven degrees of operative freedom. 
sole surgeon hand activity is emulated precisely at the surgical plane; however, da Vinci electronics intercede with beneficial motion scaling, tremor elimination, and graduated instrument tip grip. Console foot pedals control both camera $x, y$, and $z$ orientation and focus. Moreover, when the surgeon's hands engage in a clumsy position, a foot-pedal clutching mechanism allows immediate repositioning. These "eye-hand-foot" interactions enable surgeons to ratchet articulated wrists smoothly through every coordinate, configuring a myriad of complex instrument positions, while providing ergonomic comfort.

\section{Robotic Surgery Procedure Development}

An investigative protocol was created initially to develop robotic mitral valve repairs, cholecystectomies, and Nissen fundoplications. ECU Animal Care and Use Committee approval was obtained for each procedure, and humane care was used in all animal studies. Established videoscopic procedures were used as templates for development of each robotic operation. Resident surgeons were heavily involved during this development and became skilled robotic surgeons. No extramural teaching was done during this initial 6-month period. Individual procedure development was completed before using da Vinci clinically.

\section{Experimental Robotic Mitral Valve Repair}

The springboard for robotic mitral valve repair development was the endoscopic procedure spawned earlier at ECU. ${ }^{7,9,10}$ Ovine hearts best emulate human mitral valve size and configuration. Thus, 10 sheep were heparinized, euthanized, and exsanguinated. The mitral valve was exposed through a $5-\mathrm{cm}$ right minithoracotomy and left atriotomy. The right instrument port was placed in the fourth intercostal space posterior to the incision, and the left port was placed in the third intercostal space also dorsal to the incision. The three-dimensional camera was placed at the most ventrad limit of the minithoracotomy. Using da Vinci, annuloplasty bands (Edwards Life Sciences LLC, Irvine, CA) were implanted with 3-0 Ticron sutures (Tyco, U.S. Surgical Corp., Norwalk, CT). All suture placements and knot tying were done robotically. After these initial studies were complete, repairs were done in fresh human cadavers.

\section{Experimental Robotic Cholecystectomy}

Robotic cholecystectomies were performed on 20 anesthetized pigs. Robot arm port placement was similar to clinical laparoscopic cholecystectomies. Initially, the cystic duct and artery were ligated using 4-0 braided sutures. Later, an accessory port facilitated deployment of an endoscopic stapler (Tyco) for these maneuvers.

\section{Experimental Robotic Nissen Fundoplication}

Robotic Nissen fundoplications were performed in 20 anesthetized pigs. In addition to the three robotic ports

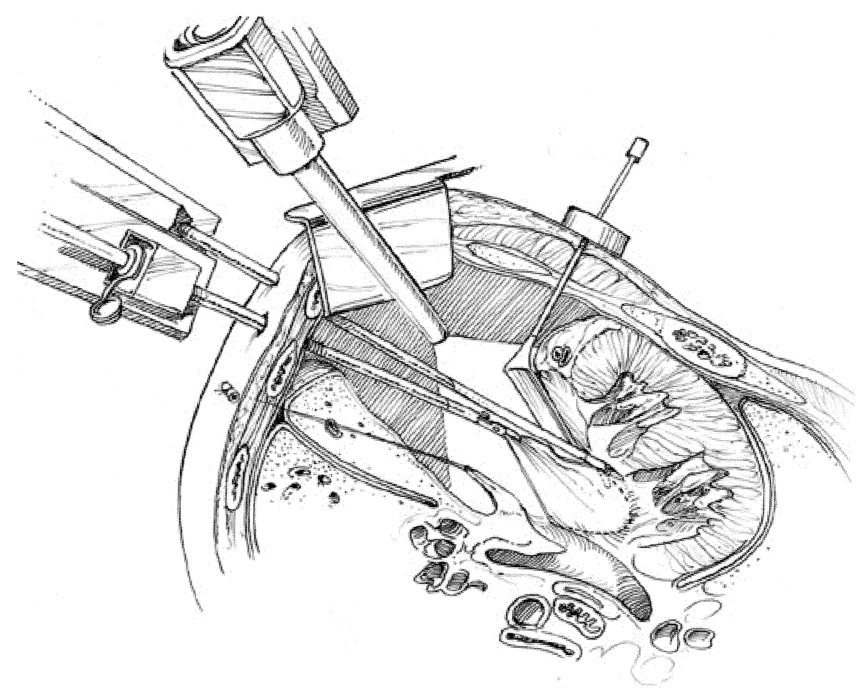

Figure 2. Cross-section of the thorax shows the position of the threedimensional videoscope as well as the intraatrial robotic arms and endowrists.

described, two accessory ports were used. One enabled insertion of a fan retractor used for visceral displacement (Tyco). An ultrasonic scalpel (Tyco) was inserted through a second accessory port to divide the short gastric vessels. The fundal wrap was done using 3-0 braided sutures. The assistant surgeon was responsible for retraction and ultrasonic scalpel control.

\section{Clinical Robotic Surgery}

After U.S. Food \& Drug Administration (FDA) approval of a mitral surgery Investigational Devices Exemption (IDE), and after full FDA approval for laparoscopic procedures, our most experienced surgeons began performing mitral valve repairs, cholecystectomies, and Nissen fundoplications clinically using da Vinci. Surgical residents participated as the patient-side assistants. For da Vinci mitral repairs, the University Health Systems of Eastern Carolina Investigational Review Board reviewed and approved an FDA-supported IDE protocol. Because da Vinci had been approved by the FDA previously for laparoscopic general surgery, approval from the Investigational Review Board was not required for clinical cholecystectomies and fundoplications. Detailed informed consent was obtained from all 52 patients before surgery. Figure 2 depicts a cross-section of the thorax, revealing the intraatrial robotic arms and wrists, and our arrangement of cardiac surgical robotic operating rooms. Figures 3 and 4 show the general surgery operating room setup for a robotic Nissen fundoplication. Detailed methods of each clinical procedure have been published recently. ${ }^{10-13}$

\section{Procedure: Data Collection and Analysis}

The following time data were collected for both experimental and clinical operations: for mitral valve repair, da 


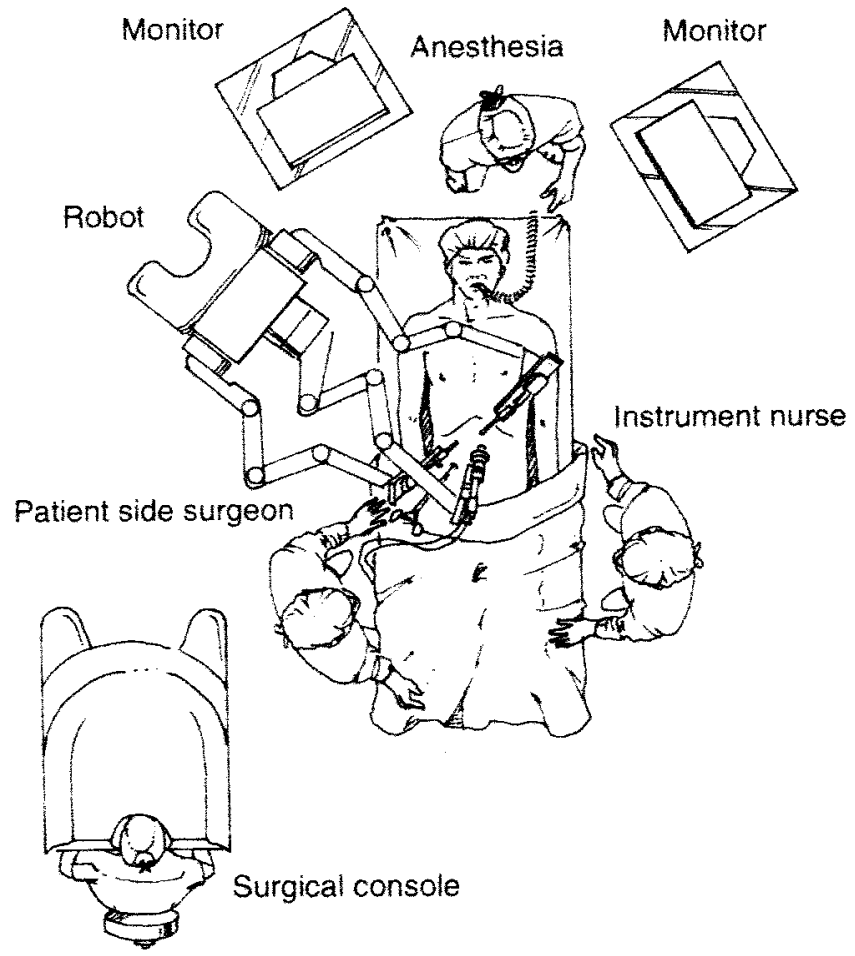

Figure 3. Setup of the operating room for general surgery procedures displays the patient-side surgical cart positioned over the patient's right shoulder.

Vinci setup, suture placement and knot tying, total annuloplasty band implantation, and the entire operation; for cholecystectomy, da Vinci setup, port placement, cystic duct and artery dissection, gallbladder dissection, and the entire operation; and for Nissen fundoplication, da Vinci setup, port placement, hiatal dissection and fundic wrapping, division of short gastric arteries, and the entire operation. Surgeon facility and instrument efficacy were documented on video for later review. All quantitative data were compared using the Student $t$ test and are presented as standard error of the mean with statistical significance shown as $P<.05$.

\section{Robotic Surgical Training Curriculum}

The FDA mandated that the device manufacturer (Intuitive Surgical, Inc.) provide comprehensive training for all institutional teams and surgeons planning to use the da Vinci surgical system clinically. In the United States, ECU was selected as the initial training site. The ECU surgical robotics training curriculum was implemented after our first clinical procedures were determined to be safe, efficacious, and facilitating. Each surgical team was trained intensively with many hands-on sessions for 2 (general surgery) or 3 (cardiac surgery) days. Surgical teams usually consisted of two surgeons (operating console and patient-side assistant) and two or three operating room nurses. Generally, cardiac teams included a senior perfusionist and in some instances an anesthesiologist. Curriculum objectives for training sur-

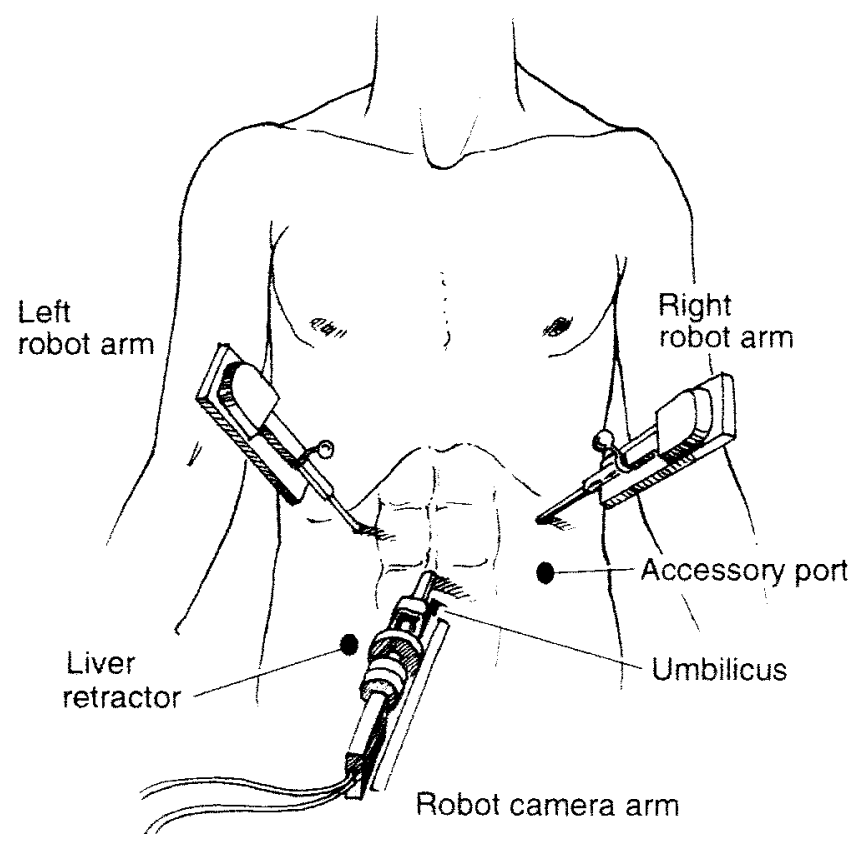

Figure 4. Performing a Nissen fundoplication requires two accessory ports in addition to the robotic instrument and camera ports. The leftsided accessory port allows delivery of the ultrasonic scalpel; the rightsided accessory port is used for retracting devices.

geons and residents were to compare surgical robotic methods with prior training and clinical experiences; to understand and master surgical robotic technology, including both electronic and mechanical components; to be able to troubleshoot all parts of the system as well as patient-robot interactions; to master surgical telemanipulation as applied to specific operations; and to become both an accomplished console-based robotic surgeon and a facile patient-side assistant.

Team curriculum objectives were similar but focused more on sterile draping, operating room arrangement, instrument interfaces with the surgical cart, and device maintenance. Teams first underwent system training that included electronic and mechanical troubleshooting as well as emergency shutdown maneuvers. After completion of system training, participants concentrated on specific procedure simulations. The curriculum advancement levels included didactic sessions, an inanimate laboratory, animal procedures, and fresh cadaver training, all completed before clinical observation (see Table 1). Because significant electrocauterization is required for cholecystectomies and Nissen fundoplications, anesthetized living animals provided feedback for hemorrhage control and prevention. For initial robotic mitral training, fresh sheep hearts were placed in a thoracic trainer. This model best emulated the geometry of the human thorax for instrument port and camera placement, while affording pliable valvular and annular tissues for suturing and repairs. Fresh cadaveric material provided the final step in hands-on mitral valve training and added the complexities associated with patient arm position and shoulder mobility. 
Table 2. DA VINCI-ASSISTED MITRAL VALVE SURGERY

\begin{tabular}{|c|c|c|c|}
\hline \multicolumn{4}{|c|}{ Experimental Mitral Valve Repairs $(n=10)$} \\
\hline & Group $1(n=5)$ & Group $2(n=5)$ & Total \\
\hline Robot setup (min) & $14.2 \pm 0.3$ & $9.6 \pm 0.5^{\star}$ & $11.9 \pm 0.8$ \\
\hline Suturing \& knot-tying (min) & $38 \pm 2$ & $31.8 \pm 0.4$ & $34.9 \pm 1.7$ \\
\hline Knot-tying (min) & $20 \pm 0.9$ & $15.2 \pm 0.5$ & $23.8 \pm 1.1$ \\
\hline Number of sutures/band & $8.6 \pm 0.2$ & $8.4 \pm 0.2$ & $8.5 \pm 0.2$ \\
\hline Total band placement (min) & $49.4 \pm 1.8$ & $41.0 \pm 0.3 \dagger$ & $45.2 \pm 1.9$ \\
\hline \multicolumn{4}{|c|}{ Clinical Robotic Mitral Valve Repairs $(n=18$ ) } \\
\hline & Group $1(n=9)$ & Group $2(n=9)$ & Total \\
\hline Draping (min) & $7.3 \pm 0.6$ & $9.1 \pm 0.4 \ddagger$ & $8.8 \pm 0.4$ \\
\hline Total robot (h) & $1.9 \pm 0.2$ & $1.8 \pm 0.2$ & $1.8 \pm 0.2$ \\
\hline Leaflet repair (min) & $56.2 \pm 8.4$ & $52.8 \pm 7.2$ & $55.3 \pm 5.5$ \\
\hline Knot-tying (min) & $2.0 \pm 0.3$ & $1.4 \pm 0.2$ & $1.9 \pm 0.1$ \\
\hline Suture and knot-tying (min) & $5.3 \pm 0.5$ & $3.6 \pm 0.1 \S$ & $4.4 \pm 0.3$ \\
\hline Number of sutures/band & $7.3 \pm 0.4$ & $9.0 \pm 0.5$ & $8.3 \pm 0.4$ \\
\hline Annuloplasty (min) & $47.4 \pm 0.2$ & $40.6 \pm 2.6$ & $44.3 \pm 2.3$ \\
\hline Aortic-clamp (h) & $2.6 \pm 0.1$ & $2.4 \pm 0.2$ & $2.6 \pm 0.1$ \\
\hline Cardiopulmonary bypass (h) & $3.4 \pm 0.2$ & $2.9 \pm 0.2$ & $3.2 \pm 0.8$ \\
\hline Skin-to-skin (h) & $5.2 \pm 0.2$ & $4.9 \pm 0.2$ & $5.1 \pm 0.8$ \\
\hline Ventilator (h) & $14.2 \pm 4.1$ & $10.0 \pm 1.0$ & $11.8 \pm 1.8$ \\
\hline ICU LOS (h) & $23.9 \pm 3.9$ & $19.3 \pm 0.8$ & $21.5 \pm 2.0$ \\
\hline Total LOS (days) & $4.6 \pm 0.5$ & $3.5 \pm 0.2$ & $3.9 \pm 0.3$ \\
\hline
\end{tabular}

ICU, intensive care unit; LOS, length of stay.

${ }^{\star} P=.001$.

$\dagger P=.03$

$\ddagger P=.046$.

$\S P=.007$

ๆ $P=.018$

\section{RESULTS}

\section{Robotic Surgical Procedures}

In the past year, ECU surgeons have developed and applied robotic technology successfully to cardiac, gallbladder, and gastric reflux surgery in 52 patients. All patients were in good general health, excepting the need for the operation specified. None of these patients had prior thoracic, cardiac, or abdominal operations. Tables 2, 3, and 4 detail both developmental and clinical results for each type of operation done with da Vinci. To evaluate procedurebased learning slopes, group 1 represents the early experience in each cohort, or the first $50 \%$ of operations, and group 2 describes the most recent half.

Table 2 depicts data analysis for robotic mitral repair studies. For laboratory studies, an average of $8.5 \pm 0.2$ sutures and $45 \pm 1.9$ minutes were required to implant annuloplasty bands. No difference was seen between group $1(\mathrm{n}=5)$ and group $2(\mathrm{n}=5)$ repairs in terms of suture placement and knot-tying times or total number of sutures. However, da Vinci setup and total band placement times were reduced significantly in group 2, indicating improved efficiency. Mitral patients $(n=18)$ were on average $56 \pm$
3 years old, and operations included quadrangular leaflet resections, chordal transfers, chordal replacements, leaflet sliding plasties, and band annuloplasties (Fig. 5). All patients had band annuloplasties, and all but one had a leaflet and/or chordal procedure. An average of $8.5 \pm 0.4$ sutures were placed in each band, requiring $4.4 \pm 0.3$ minutes per suture and knot. In group 2 patients, significantly decreased times were required for robot draping and suturing and knot tying. Interestingly, in group 2 patients, significantly more sutures were placed per band, with less time required. Times for cardiopulmonary perfusion and ventilator dependency as well as intensive care unit and hospital length of stays appeared to be reduced in group 2 patients but never reached statistical significance. The group 2 patients were discharged after approximately $3.5 \pm 0.2$ postoperative days versus $4.6 \pm 0.2$ days in group 1 patients. Later repairs were increasingly more complex, with fluctuating individual procedure times in each cohort. On repair completion, one patient had mild regurgitation by intraoperative transesophageal echocardiogram.

Table 3 shows developmental and clinical data comparisons for the cholecystectomy procedures. Group 2 experimental procedures showed time decreases in all components 
Table 3. DA VINCI-ASSISTED CHOLECYSTECTOMY

\begin{tabular}{|c|c|c|c|}
\hline \multicolumn{4}{|c|}{ Experimental Cholecystectomies $(n=20)$} \\
\hline & Group $1(n=10)$ & Group $2(n=10)$ & Total \\
\hline Robot setup time (min) & $19.1 \pm 0.5$ & $10.6 \pm 0.6^{\star}$ & $14.5 \pm 1.1$ \\
\hline Port placement (min) & $14.0 \pm 0.5$ & $9.0 \pm 0.2^{*}$ & $11.5 \pm 0.7$ \\
\hline Duct/artery dissection (min) & $15.2 \pm 0.7$ & $7.6 \pm 0.4^{*}$ & $11.4 \pm 1.0$ \\
\hline Gallbladder dissection (min) & $17.1 \pm 0.4$ & $9.0 \pm 0.2^{*}$ & $13.0 \pm 1.0$ \\
\hline Total procedure time (min) & $65.3 \pm 1.6$ & $36.8 \pm 0.9^{\star}$ & $51.0 \pm 3.5$ \\
\hline \multicolumn{4}{|c|}{ Clinical Cholecystectomies $(n=20)$} \\
\hline & Group $1(n=10)$ & Group $2(n=10)$ & Total \\
\hline Body mass index & $29.3 \pm 2.4$ & $28.8 \pm 1.8$ & $28.4 \pm 1.2$ \\
\hline Age $(y r)$ & $48.8 \pm 6.4$ & $35.0 \pm 7.0$ & $40.6 \pm 3.9$ \\
\hline Robot setup time (min) & $18.1 \pm 1.1$ & $16.5 \pm 0.8$ & $17.3 \pm 0.9$ \\
\hline Duct/artery dissection (min) & $9.2 \pm 1.1$ & $4.0 \pm 0.6 \dagger$ & $6.9 \pm 0.8$ \\
\hline Gallbladder dissection (min) & $10.4 \pm 3.1$ & $5.0 \pm 0.8$ & $7.0 \pm 1.4$ \\
\hline Total robot time (min) & $38.8 \pm 3.3$ & $21.2 \pm 3.0 \neq$ & $28.5 \pm 2.7$ \\
\hline Total procedure time (min) & $68.6 \pm 3.5$ & $52.4 \pm 3.3 \S$ & $62.2 \pm 3.2$ \\
\hline \multicolumn{4}{|l|}{$\begin{array}{l}{ }^{\star} P<.05 . \\
\dagger P=.02 . \\
\ddagger P=.01 \\
\S P=.006 .\end{array}$} \\
\hline
\end{tabular}

of the procedure, suggesting that the operator's skill improved with robotic experience. This is not unexpected in less variable experimental robotic operations. The da Vinci cholecystectomy patients $(n=20)$ averaged $40.6 \pm 3.9$ years of age, and none had acute cholecystitis. Total series surgical times were reasonable at $62.2 \pm 3.2$ minutes but decreased significantly by approximately 16 minutes between group 1 and 2 patients (group $2=52.4 \pm 3.3$ minutes). Similarly, cystic duct/artery dissection and total robot time decreased between groups 1 and 2. This decrease in surgical time is encouraging and probably resulted from the combination of robotic experience and use of the surgical stapling device.

Table 4 shows statistical comparisons for comparative Nissen fundoplication cohorts. Figure 6 shows a robotic fundal wrap and suture approximation for this procedure. Group 2 experimental operations showed significant time reductions for port placement, hiatal dissection/fundic wrapping, and short gastric artery division/ligation. Moreover, total procedure time was significantly reduced by about 36 minutes. For robotic Nissen fundoplication patients $(\mathrm{n}=14)$, the mean age was $49.6 \pm 5.6$ years. Times for port placement, hiatal dissection, fundic wrapping, short gastric division, and fundal/crural suturing times tended to decrease in group 2, but the difference was not significant. However, compared with group 1, robot use in group 2 (107.2 \pm 3.2 minutes) Nissen patients decreased by about 25 minutes, and the total procedure time decreased by approximately 37 minutes. Thus, as the complexity of our surgical procedures increased, robotic facilitation improved, resulting quickly in surgical times similar to standard laparoscopic techniques.

\section{Robotic Surgical Training}

In 9 months (August 2000 through April 2001), 75 attending surgeons, 15 residents, 31 nurses, and 30 surgical teams have completed the ECU training curriculum (see Table 1). All teams trained on da Vinci have completed successful clinical procedures in their institutions with the robotic system. The cardiac surgeons completing the curriculum have begun an 11-center FDA Robotic Mitral Valve Repair Trial, which will enroll more than 100 patients for institutional comparisons. To date, quantitative evaluation of surgical dexterity and efficiency has not been possible; however, proctor observation and self-testing suggested that each previously experienced surgeon gained significant da Vinci system knowledge and would be able to use the device in clinical cases competently. Surgical team education directed toward technologic cohesion was perhaps the most important aspect of the training, and each team was very able by the end of the course.

\section{DISCUSSION}

The widespread acceptance of endoscopic general surgery was heralded by independent work by Mouret, DuBois, and Reddick, who performed the first laparoscopic cholecystectomies in the mid-1980s. Despite early skeptics, more than 15,000 general surgeons were trained in these video- 
Table 4. DA VINCI-ASSISTED NISSEN FUNDOPLICATION

\begin{tabular}{|c|c|c|c|}
\hline \multicolumn{4}{|c|}{ Experimental Nissen Fundoplications $(n=20)$} \\
\hline & Group $1(n=10)$ & Group $2(n=10)$ & Total \\
\hline Robot setup time (min) & $18.3 \pm 0.7$ & $17.0 \pm 0.8$ & $17.7 \pm 0.7$ \\
\hline Port placement (min) & $15.5 \pm 0.9$ & $9.4 \pm 0.9^{*}$ & $12.3 \pm 1.0$ \\
\hline Hiatal dissection/wrap (min) & $31.8 \pm 0.7$ & $9.8 \pm 1.2 \dagger$ & $20.8 \pm 2.8$ \\
\hline Short gastrics division (min) & $13.5 \pm 0.5$ & $5.5 \pm 0.2$ & $9.5 \pm 1.1$ \\
\hline Suture placement/tying (min) & $17.2 \pm 1.6$ & $19.0 \pm 0.8 \neq$ & $18.0 \pm 1.2$ \\
\hline Total procedure time (min) & $86.7 \pm 2.9$ & $51 \pm 14.3 \ddagger$ & $66.3 \pm 5.5$ \\
\hline \multicolumn{4}{|c|}{ Clinical Nissen Fundoplications $(n=14)$} \\
\hline & Group $1(n=7)$ & Group $2(n=7)$ & Total \\
\hline Body mass index & $29.1 \pm 1.1$ & $30.8 \pm 1.1$ & $29.6 \pm 1.1$ \\
\hline Age (yr) & $51.8 \pm 4.5$ & $50 \pm 5.8$ & $49.6 \pm 5.6$ \\
\hline Robot setup time (min) & $18.3 \pm 1.3$ & $17 \pm 0.9$ & $17.5 \pm 1.0$ \\
\hline Port placement (min) & $15.7 \pm 4.2$ & $10.4 \pm 4.1$ & $13.5 \pm 4.9$ \\
\hline Hiatal dissection/wrap (min) & $25.1 \pm 4.6$ & $14.4 \pm 2.5$ & $19.7 \pm 3.1$ \\
\hline Short gastrics division (min) & $14.0 \pm 2.9$ & $10.4 \pm 1.0$ & $12.0 \pm 1.8$ \\
\hline Crural sutures (min) & $4.8 \pm 0.4$ & $4.6 \pm 0.3$ & $4.6 \pm 0.4$ \\
\hline Fundal sutures (min) & $6.4 \pm 1.1$ & $4.4 \pm 0.4$ & $5.2 \pm 0.8$ \\
\hline Total robot time (min) & $88 \pm 5.5$ & $63.4 \pm 4.3 \S$ & $73.4 \pm 4.5$ \\
\hline Total procedure time (min) & $144 \pm 11.3$ & $107.2 \pm 3.2 \rrbracket$ & $121.4 \pm 3.3$ \\
\hline
\end{tabular}

\footnotetext{
${ }^{*} P=.06$.

$\dagger P=.001$

$\ddagger P=.04$.

$\S P=.01$.

१ $P=.03$.
}

techniques between 1990 and $1992 .{ }^{14-16}$ Since then, advanced laparoscopic surgery has become the mainstay of most general surgical practices. Recently, Mohr, ${ }^{17}$ Reichenspurner, ${ }^{18}$ Vanermen, ${ }^{19}$ and Chitwood ${ }^{20}$ have expanded the use of assisted vision to cardiac surgery. These surgeons have shown excellent, reproducible results when repairing complex mitral valve lesions using assisted vision and tiny thoracic incisions. Nevertheless, the limitations of twodimensional endoscopy and long, motion-amplifying instruments has dampened most cardiac surgeons' enthusiasm for scaling this technologic ascent.

The expanded future of minimally invasive surgery resides in the surgeon's ability to extend operational facility beyond direct hand contact and to reach more difficult surgical targets with greater dexterity and comfort. Krummel and Mack recently projected that in the near future, surgical vision and training systems will be able to model most surgical operations through immersive technology. ${ }^{21-24}$ They have suggested the probability of developing training consoles that can emulate hemorrhage, skin turgor, and even organ reality. However, significant electronic and mechanical improvements are needed to expand surgical indications and enable true "nano-surgery," including fetal, pediatric, and congenital heart applications. Current robotic surgical systems provide articulated instrument wrists with seven degrees of motion freedom, tremor filtration, motion scaling, graded tip-effector grasping abilities, and ergonomic hand motions. Tactile feedback is far from perfect, although gross instrument collisions and obstructions can be sensed. These new instrument capabilities become additive with high-resolution three-dimensional vision to entrain the surgical field, enabling precise intracavitary telemanipulation. Because visual cues provide more than $70 \%$ of human sensory input, optical magnification and high-resolution cameras with macro-zoom capabilities go a long way toward compensating for haptic loss. ${ }^{21}$ Thus, many of the obstacles to traditional endoscopic surgery can be avoided, including image inversion, long-instrument tremor amplification, and range-of-motion limitations.

To perform operations in restricted spaces, surgeons will need to increase facilitation through increasing instrument design. Generally, surgeons prefer to establish serial "comfort zones" before progressing to more advanced technical levels. Most young surgeons experienced laparoendoscopic or video-assisted thoracic training in their residencies. However, gaining experience in robotic surgery differs greatly. Heretofore, most endoscopic operations have been ablative or extirpative rather than reconstructive. Cardiac surgeons have not embraced videoscopic methods owing to surgical complexity and safety. Thus, among various specialties and between surgeons, experience and training fields remain unlevel today. 

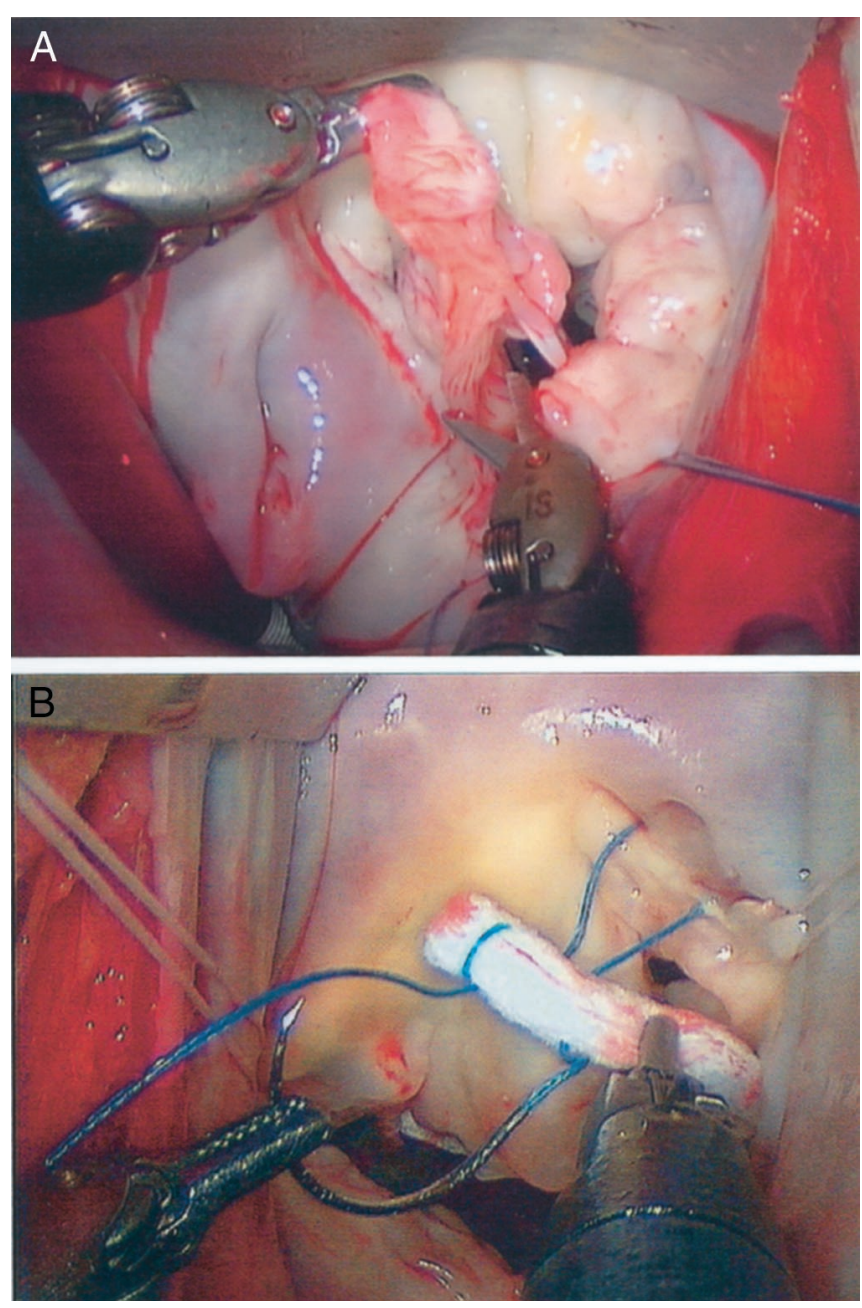

Figure 5. Magnified three-dimensional vision provides improved visualization of valvular structures. (A) A prolapsing segment of the posterior mitral leaflet is excised using tissue scissors and tissue forceps in the right hand and left hand, respectively. (B) An annuloplasty band is secured to complete the mitral repair procedure. Two needle holders are used to place sutures through the annulus. Computer assistance allows the surgeon to be ambidextrous in placing sutures around the annulus.

Prototypic robotic devices first were used in Europe in 1997 for abdominal surgery and in 1998 for mitral valve and coronary operations. ${ }^{25-28}$ In the United States, clinical trials have just begun to evolve. ${ }^{29}$ For these early robotic surgeons, no formal training has been available, and they learned by doing new operations in an unfamiliar milieu with more complex instruments. These early successes forecasted promise for robotic applications in complex reconstructive and anastomotic surgery. The first FDA robotic mitral trial was approved in 1999 and began in May 2000. ${ }^{11}$ Thereafter, the FDA mandated that surgical teams and surgeons undergo formal training before beginning clinical work. The da Vinci device was approved for general use in laparoscopic surgery and thoracoscopy in 2000 and 2001. Our curriculum has been used to train 30 surgical teams in general, cardiac, and urologic surgery. Most of these surgeons have focused on mitral repair, gallbladder, and gastric
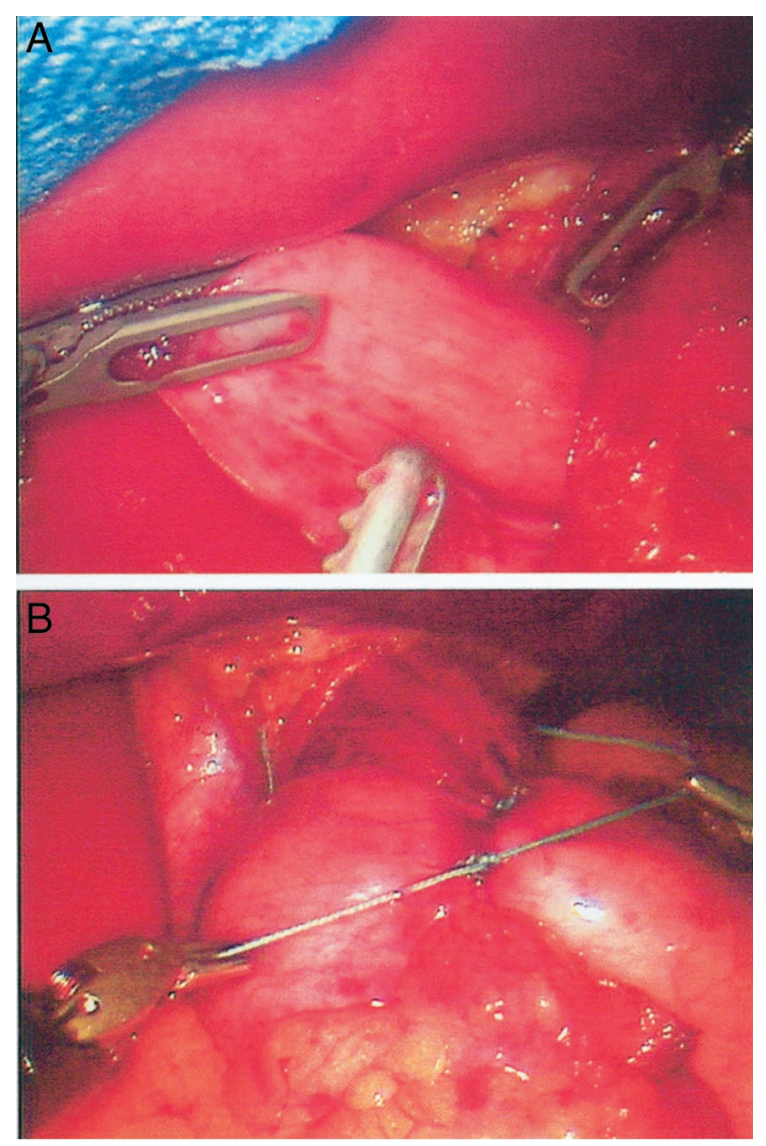

Figure 6. A Nissen fundoplication is performed. (A) Cadiere forceps are used to execute the fundal wrap procedure. (B) Sutures are used to secure the wrap around the esophagus just as in conventional or laparoscopic procedures.

reflux surgery; however, techniques for internal thoracic artery harvesting as well as adrenalectomies and prostatectomies have been taught. We are developing similar educational programs for gastric bypass and gynecologic operations. Our group has recently performed adrenalectomies and splenectomies using the robot without complications. ${ }^{30,31}$

A structured plan for procedure development and training seems to be the "launch pad" required to optimize early results. The ECU multispecialty surgical robotic training program offers structured, intense robotic training, specialty cross-pollination, and the closest clinical simulation available today. Our objective-based curriculum is structured so that learners can master the details of robotic surgical systems and facilitate intraoperative troubleshooting. Surgeons selected for the curriculum were experienced general and cardiac surgeons, and all were facile technical specialists. The course did not emphasize indications or perioperative management. Interestingly, resident surgeons' skills in the laboratory were gained as fast as those of experienced surgeons. To date, resident surgeons have provided mostly patient-side assistance, but our training program is becoming more directed toward console experience. 
Data derived from our experimental and clinical studies suggest that teledirected cholecystectomies, Nissen fundoplications, and mitral valve repairs, performed with the da Vinci surgical robot, can be safe, efficacious, and accurate. These results show that robotic surgical times should decrease with a relatively short experience. Hospital lengths of stay approximated those of traditional laparoscopic operations but were shorter than for conventional mitral surgery. Patients were discharged after 4.5, 1.0, and 0.9 days for mitral valve repairs, fundoplications, and cholecystectomies, respectively.

This work describes the first U.S. experience of robotic mitral valve repairs performed robotically. Clinical results in patients were excellent, with no residual valve leakage or deaths. Although for the later group 2 mitral patients, cardiopulmonary bypass time, total surgical time, ventilator time, and intensive care unit and hospital length of stay were statistically similar to the first cases, a strong trend toward time reduction was evident throughout (see Table 2). For experimental and clinical cholecystectomies, tissue dissection time, robotic time, and total procedure time decreased significantly for the same periods, and telemanipulation lasted only 21 minutes for later cases. Times to complete robotic Nissen fundoplications decreased markedly in both experimental and clinical groups. In the later group, the robotic time was reduced to 63 minutes, with a procedure time of 107 minutes (see Table 4). These surgical times show parallel improvement pathways despite the particular robotic procedure. Development pathways for robotic surgical technology seem to be inscribing an arc similar to that of the "new technology" of the 1980s.

Multispecialty microsurgery should enjoy a greater potential with the advent of robotic technology. After proper academic training, operations of the same or better quality can be performed with these new devices, and procedure times should decrease with proctored experience. Moreover, we have shown that intensive care unit and hospital lengths of stay and complication rates are the same as for standard endoscopic operations. No doubt, surgical pathway memory, to teach experienced learners and residents alike, would be of great benefit. Also, virtual operations could be based on digital vision data from actual operations. Radiographic instrument positioning and navigation systems appear to be the next technology to be compounded with surgical robotics. Placement of ports and cameras with increasing accuracy and development of smaller instruments and telescopic "end-chip" three-dimensional cameras will carry new-era surgeons closer to a robotic-assisted surgery reality. Of course, large comparative patient series are needed for this determination.

\section{References}

1. Bonchek LI, Ullyot DJ. Minimally invasive coronary bypass: a dissenting opinion. Circulation 1998; 98:495-497.
2. Lytle BW. Minimally invasive cardiac surgery [editorial; comment]. J Thorac Cardiovasc Surg 1996; 113:554-555.

3. Cooley DA. Antagonist's view of minimally invasive heart valve surgery. J Card Surg 2000; 15:3-5.

4. Felger J, Chitwood WR Jr, Nifong LW, et al. Evolution of mitral valve surgery: toward a totally endoscopic approach. Ann Thorac Surg (in press)

5. Felger J, Nifong LW, Chitwood WR Jr. Robotic valvular surgery. Curr Opinion Cardiol 2001; 15:146-151.

6. Chitwood WR Jr. State-of-the-art review: videoscopic minimally invasive mitral valve surgery. Trekking to a totally endoscopic operation. Heart Surg Forum 1998; 1:13-16.

7. Chitwood WR. Video-assisted and robotic mitral valve surgery: toward an endoscopic surgery. Sem Thorac Cardiovasc Surg 1999; 11:194205.

8. Chitwood, WR. Video-assisted mitral valve surgery: using the Chitwood clamp. Oper Tech Thor Cardiovasc Surg 2000; 5:190-203.

9. Falk V, Autschbach R, Krakor R, et al. Computer-enhanced mitral valve surgery: toward a total endoscopic procedure. Semin Thorac Cardiovasc Surg 1999; 11:244-249.

10. Chitwood WR, Elbeery JR, Chapman WHH, et al. Video-assisted minimally invasive mitral valve surgery: the "Micro-Mitral" operation. J Thorac Cardiovasc Surg 1997; 113:413-414.

11. Chitwood WR Jr, Nifong LW, Elbeery JE, et al. Robotic mitral valve repair: trapezoidal resection and prosthetic annuloplasty with the da Vinci surgical system. J Thorac Cardiovasc Surg 2000; 120:11711172.

12. Kim VB, Chapman WHH III, Albrecht RJ, et al. Early experience with telemanipulative robot-assisted laparoscopic cholecystectomy using da Vinci. Surg Laparosc Endosc Percutaneous Tech (in press).

13. Chapman WHH III, Young JA, Albrecht RJ, et al. Robotic Nissen fundoplication: alternative surgical technique for the treatment of gastroesophageal reflux disease. J Laparoendoscopic Adv Surg Tech 2001; 11(pt A):27-30.

14. Litynski GS. Profiles in laparoscopy: Mouret, Dubois, and Perissat: the laparoscopic breakthrough in Europe (1987-1988). JSLS 1999; 3:163167.

15. Reddick EJ, Olsen DO. Laparascopic laser cholecystectomy: a comparison with minilap cholecystectomy. Surg Endosc 1989; 3:131-133.

16. Soper NJ, Brunt LM, Kerbl K. Medical progress: laparoscopic general surgery. N Engl J Med 1994; 330:409-419.

17. Mohr FW, Falk V, Diegeler A, et al. Minimally invasive port-access mitral valve surgery. J Thorac Cardiovasc Surg 1998; 115:567-574.

18. Reichenspurner H, Boehm DH, Welz A, et al. Three-dimensional video and robot-assisted port-access mitral valve surgery. Ann Thorac Surg 2000; 69:1176-1181.

19. Vanermen H, Wellens F, De Geest R, et al. Video-assisted port-access mitral valve surgery: from debut to routine surgery. Will trocar-portaccess cardiac surgery ultimately lead to robotic cardiac surgery? Semin Thorac Cardiovasc Surg 1999; 11:223-234.

20. Chitwood WR Jr, Nifong LW. Minimally invasive videoscopic mitral valve surgery: the current role of surgical robotics. J Cardiac Surg 2000; 15:61-75.

21. Meir AH, Rawn CL, Krummel TM. Virtual reality: surgical application-challenge for the new millennium. J Am Coll Surg 2001; 192:372-384.

22. Mack MJ. Minimally invasive and robotic surgery. JAMA 2000; 285:568-572.

23. Gorman PJ, Meir AH, Krummel TM. Simulation and virtual reality in surgical education: real or unreal. Arch Surg 1999; 134:1203-1208.

24. Gorman AJ, Meir AH, Krummel TM. Computer-assisted training and learning in surgery. Comput Aided Surg 2000; 5:120-130.

25. Himpens J, Leman G, Cadiere GB. Telesurgical laparoscopic cholecystectomy. Surg Endosc 1998; 12:1091.

26. Altschulbach R, Onnasch JF, Falk V, et al. The Leipzig experience with robotic valve surgery. J Cardiac Surg 2000; 15:82-87. 
27. Loulmet D, Carpentier A, d'Attelis N, et al. Endoscopic coronary artery bypass grafting with aid of computer-assisted instruments. J Thorac Cardiovasc Surg 1999; 48:4-10.

28. Reichenspurner H, Boehm DH, Gulbins H, et al. Robotically assisted endoscopic coronary artery bypass procedures without cardiopulmonary bypass. J Thorac Cardiovasc Surg 1999; 118:960-961.

29. Damiano RJ, Tabaie HA, Mack MJ, et al. Initial prospective multicenter clinical trial of robotically assisted coronary artery bypass grafting. Ann Thorac Surg (in press).

30. Young JA, Chapman WHH III, Kim VB, et al. Robotic assisted adrenalectomy for a rare adrenal incidentaloma: case in review. Surg Laparosc Endosc Percutaneous Tech (in press).

31. Chapman WHH III, Albrecht RJ, Kim VB, et al. Computer assisted laparoscopic splenectomy with the da Vinci surgical robot. J Laparoendosc Adv Surg Tech (in press).

\section{Discussion}

Dr. Thomas M. Krummel (Stanford, California): Congratulations to Dr Chitwood and his group for this very first presentation of surgical robotics at the American Surgical. There are many things to discuss. I would like to focus in particular on the generic problem of assimilating new technologies into surgical practice.

If you think about today's program, how many times have we heard 'in experienced hands" or 'the learning curve is steep'? However, seldom have we as a profession considered the problem that that experience or that learning curve occurs on patients. There was a very tangible demonstration of that during the early days of laparoscopic cholecystectomy. Common duct injury rates transiently increased by almost 10-fold; patients paid the price for our learning curve.

If we think about it, sports are practiced, music is practiced, aviation is practiced. What Dr. Chitwood and his colleagues have now demonstrated, is that surgical robotic technique can be practiced. No more see-one, do-one, teach-one. Parenthetically, it is interesting that it was the FDA that mandated this training, not one of our surgical organizations.

While surgical practice and skills assessment seem intuitive, the impediments are formidable: Space. Money. Time. Tradition. Thus, four questions, Dr. Chitwood.

One, how did you deal with the space for a training facility? There is no indirect cost recovery for such a laboratory.

Second, training facilities are expensive to build and operate, and a surgical robot costs about a million dollars. Any suggestions for approaching our respective CFOs?

Third, as resident and faculty time is scarce at best, how do you integrate a scheduled additional extracurricular activity into the typical frantic day or week?

Finally, I think most of us would agree that our surgical residencies are educational programs and not forced labor camps. However, the tradition of service is enormous. How do you deal with the faculty member who wants 'their resident' available any place, anytime, when in point of fact that resident may be in a learning laboratory practicing surgery?

Presenter Dr. W. Randolph Chitwood, JR. (Greenville, North Carolina): Thank you very much, Dr. Krummel. Dr. Krummel has pioneered much of the work in virtual reality learning and this type of work and has written quite extensively in this area.

So I think that one comment that is appropriate, that basically we should not risk patients for this new technology. This technology should carry us to the new level of learning so that we are risking a virtual image but not a patient to learn. They don't land a 747 the first time in the real plane.

To answer your question regarding space, space is a problem. We have not had that problem in that being chairman of surgery does help to set the goals for the department. And also, when we have the opportunity to get space, we jump at the chance. We basically included this in our new facility as we built it and built the robotic lab before we had the robot.

The cost of the robot, about a million dollars. The way we handled this, we had a very enlightened CEO of the hospital who felt that this was not going to be a cost recovery item, no business plan for this, but it is something we should do to develop the technology and to put something back.

On the medical school side, the department split the cost with the dean. Since we had given many deans lots of dollars in the past, we thought that would be fine. So we were able to fund this. But many people have to fund this from extramural funds from philanthropic organizations but not from clinical practice.

How do we integrate the attending time in the operating room? A complex question. You have to have people who want to do it. There also has to be intellectual curiosity. And I have several individuals who have that. You will do stuff you like to do. No matter how tired you are, you will go play with the robot. Dr. Pellegrini was down at our place and played with the robot. Everybody, even the dean, likes to sit at the robot. So there is no trouble getting our clinical faculty to find the time to work, even in the laboratory.

How do we handle it in the operating room? We make it easy for them in that we don't have a dedicated operating room for robotic surgery. We move the robot where the surgery is to be done. We are very close between our general and cardiac rooms so we are able to share the device and schedule it. And we are doing cases every week now. We are up to, I think, about 23 mitral valves.

The residents, we don't let them be forced labor in that basically Dr. Pories, who was chairman of our department for many years, realized very early that really they are there to train residents. We have to have other individuals to help us do the hard work, the scut work. So we have physician extenders with dedicated residents. If you look at the manuscript, there will be about six residents on that paper who worked very hard, nights, days, time off, probably past normal working hours.

Dr. Ralph J. Damiano (St. Louis, Missouri): I would first like to congratulate Dr. Chitwood and his team for their pioneering work in this area both in terms of procedure development and in focusing attention on the very important issue of surgical training. At our institution, we have used the Computer Motion system. There is similar requirements from the FDA requiring training, so I know what a difficult issue this is for investigators

The real question behind this is when is a surgeon ready to do a new operation or use a new technology? As Dr. Krummel suggested, this is an extremely difficult question to answer, and one that we have not particularly addressed well in the history of our profession. Unfortunately, our learning curve has traditionally been performed on patients. One of the strengths of this new technology is it may allow for important training and practice out of the operating room in either a simulated environment or using the type of trainers Dr. Chitwood has described in his talk.

I have several questions for you regarding training with this technology.

First of all, how do you know when the skill or procedure has been learned by these teams? Have you developed objective criteria for when the team is ready to do their first patient? Is this a minimum number of hours of practice? Do you look for a plateau in the learning curve or do you have certain objective criteria, i.e. a certain time required to perform either a simple or complex task?

My second question is whether you have developed specific drills to assess surgical ability with the robotic system? And do you think these drills are different for someone trying to learn a laparoscopic Nissen versus a mitral valve repair?

Have you performed any evaluation to see what the real benefits of robotics are over traditional laparoscopy or endoscopy? As you know, Dr. Gagner and his group first at the Cleveland Clinic and now at Mount Sinai have shown that there were no benefits of robotics over laparoscopy with either simple or complex motor skills.

At Washington University, we have just recently finished a randomized trial of laparoscopic versus robotic surgical techniques in a carefully designed set of drills. In every one of the drills, robotics were slower than laparoscopic, land-held instruments. However, we did notice that the precision was increased, particularly in complex motor drills. I wonder if you have any comments on this issue.

DR. W. RANDOlPh Chitwood, JR.: Thank you very much, Dr. Damiano. 
Dr. Damiano is also another person who has worked very hard to see if this is the real thing through his work with coronary surgery. He did the first coronary anastomosis with a robot in the United States several years ago.

How do you know what they have learned? Well, it is very difficult. That is next part of our laboratory, to develop specific methods and training paradigms that will provide us with a number, a quantitative number, to say ready or not.

I will make one comment, that I think many of these individuals, whether they are experienced laparoscopic surgeons or cardiac surgeons, who really never had any interest or work in videoscopic surgery, when they come through the tunnel it normalizes everyone. Because laparoscopic surgeons, who have a lot of talent and do many things, are taken down to a learning curve that they have to accept.

So to answer your question, it is still pretty qualitative. I would tell somebody if I don't think they are ready. But the team assessment is key. Because generally you can have an excellent surgeon who can do all the manipulations with the device, but if the team can't do the exchanges of the instruments, troubleshooting - it is a real team effort - then it all goes for naught.

So we emphasize team training. We don't like to train people who just do the surgery. We like to bring the nurses, the perfusonists, and possibly the anesthesiologists, and put them through the inanimate training, the animal training, observation and cadaver training. We think this is the best we have today.

What are the benefits of robotics versus laparoscopic surgery? I really can't tell you. I know that the Wright Brothers when they came to North Carolina in 1909 didn't have a very good model, but by 1920 they could fly pretty well. So I think today when you compare these side by side, you won't get the advanced results that you would like to see. The patient benefits are the same as far as feeling well, but it does facilitate the surgeon's ability.

Let us drift ten years, where you have 3 millimeter cameras with chips at the end that totally immerse you, no camera problems, no spherical aberration, or, very small instruments, nano technology, for these robotic arms. You can do even fetal heart surgery and develop new types of operations that we have not been able to do before. I don't want to be a zealot, but as it develops I think we have to look toward the new technology that will carry us to a difference between the two.

Dr. William A. Baumgartner (Baltimore, Maryland): Dr. Chitwood and his colleagues have been pioneers in this area of surgery. I think the emphasis though is on the word 'development," as this device is clearly in its infancy. In future years, however, it will make a variety of operations in various subspecialties truly minimally invasive.

Our own robotic system at Hopkins has been used primarily in general surgery as we await our final IRB approval to start the mitral valve surgery program. Our surgeons are some of the data elements in Dr. Chitwood's paper. At Hopkins, Dr. Mark Talamini has done over 60 procedures using the intuitive robot, predominantly Nissen fundoplications but also some cholecystectomies as well as exploratory laparotomies and bowel resections. He clearly prefers to use this intuitive system over standard endoscopic techniques.

However, in order for this system to significantly advance, there has to be concurrent development of other technical aspects associated with the robot. Dr. Chitwood, would you please comment on the following issues: improved visualization; better anastomotic techniques, such as the automatic stapling device; and advances in hepatic technology which will allow appropriate sensory feedback.

Lastly, as has been mentioned by Dr. Krummel as well as Dr. Damiano, is the issue of residency training. How do you envision this occurring in robotic surgery? Will it be as you have instructed and shown us here and by laboratory simulation? Or, rather, is there a role for having two consoles in the operating room to allow participation of an assistant surgeon similar to how we teach today?

You have applied this technique and helped to define its evolution in the field of surgery at East Carolina University. Thank you for this excellent update.
Dr. W. Randolph Chitwood, JR.: Thank you very much, Dr. Baumgartner. I certainly appreciate you having your institution enrolled in the robotic system trial for mitral valve surgery.

You asked a very important question regarding adjunctive or concurrent technology. This is what will bring a lot of this to fruition, through navigation systems, and in the instances of heart surgery, cardiopositioning systems, where basically you will know either from MRI or echo exactly where to place the trocars in the heart. Whether there is a holographic image overlay that where basically you can see exactly where the organ is in position, the HIP NAV technology for orthopedic surgery or the stealth technology for neurosurgery, the lesion and you can vector peripherally in on this. Show Peripheral position is one of the more difficult problems. Visualization improvements are only in the beginning now, and will develop markedly in the next several years.

We are using coalescent clips, yet. Hepatic technology is far away. But as Dr. Krummel wrote in one of his papers, really only 8 to $10 \%$ of our sensory system is tactile, 70 to $80 \%$ is from our visual input. So I think visual cues are absolutely the most important. Also, accessory arms can come in positioning retractors. So adjunctive technology will be very important.

The concept about two consoles I think is very clearly a potential. Perhaps a little pilot console, not the full console but just the control box like my computer here. Basically it is like driver's ed, you can put your foot on the brake, or you can take that stitch. You can digitize data from an operation and create the virtual operation, which is really the operation just done but it is in a model that the resident can recreate. That is clearly possible. A lot of people tell me it can't be done, but I will bet Dreamworks could do it today.

Dr. Timothy J. Gardner (Philadelphia, Pennsylvania): I agree with Dr. Krummel that perhaps the most important aspect of this presentation is that this may be the first time that the American Surgical Association has heard about robotic surgery. Although it seems rather unlikely that robotic techniques will be widely adopted any time soon, this work represents an important first step, as has Ralph Damiano's work as well.

There are, however, all sorts of issues, not just the training of surgeons in these techniques, but also the financing of such high technology efforts. Although it is very helpful to have individuals like Dr. Chitwood, who has control over his practice environment, it can be extremely difficult in this era of financial constraints for health care to obtain the kind of support needed for these types of efforts.

We need the collaboration, the R\&D efforts and the financing of industry. We also need to get NIH's attention for such investigations. The biomedical research community must understand that some of the advancements that will bring medicine forward to the next level are not all going to be at the molecular level but are going to involve biomechanics, new technology and new techniques. Another major challenge will be to convince HCFA that the increased costs associated with such new technology will be worth investing in.

We have a lot of issues to deal with as we move forward, and it is fortuitous to have a few zealot innovators like Dr. Chitwood to keep us focused in the future.

Dr. W. Randolph Chitwood, JR.: Please, don't call me a zealot, Dr. Gardner.

I asked Dr. Gardner to discuss the paper because he has always been my conscience. He is always kept me straight and given me good advice. His questions are absolutely right on target.

I think financing is a real problem. We do have to collaborate with industry. Also, I think the NIH is where we have got to go for funding. Because material properties are not iterating as fast as the electronic properties and the electronics changes. How do we make robotic arms very, very small if we don't have the biomechanical properties to do this? New types of metals? Much of this could be done through basic research, which needs funding.

I think we have to again engage politicians, engage HCFA. We should realize that the cost of this will come down some but it is not going to be $\$ 50$ a case. I think that the patients will vote with their feet if it really is 
good technology. But we must do a better job of evaluating this ourselves, and making sure our patients are well protected. If they are well protected, they won't vote with just their feet, they will vote with their votes.

Dr. Mark A. TAlamini (Baltimore, Maryland): I also congratulate Dr. Chitwood and his group for pushing this exciting field forward. I first encountered the da Vinci system as one of the FDA panel members that mandated all of this additional training. Since obtaining our system, I have become rapidly spoiled by the excellent visualization and the fine instrument control that it provides.

My question is similar to Dr. Baumgartner's. The difference between these operations and open or laparoscopic operations is that the attending surgeon doing the latter types of operations can completely control things by moving tissues out of the way laparoscopically or grabbing the resident's hands during an open operation. The surgeon sitting at the console during these operations is the only one who has total control. So until we have a driver's ed type of situation, I would like to ask how you think we can deal with that situation.

DR. W. RandolPh Chitwood, JR.: Thank you very much, Dr. Talamini. Again, Dr. Talamini is a pioneer in all of this work and should be given credit for great advancements in general surgery with this device.

We have integrated our residents from the very beginning, working on the papers, working on the development, in the operating room and patient safety. Resident education should be our highest goal. New era residents are often better than their teachers. 